\title{
Prevalence of and factors associated with dysfunctional low back pain in patients with rheumatoid arthritis
}

\author{
Kazuto Miura ${ }^{1}$ - Osamu Morita ${ }^{1} \cdot$ Toru Hirano $^{2} \cdot$ Kei Watanabe $^{2} \cdot$ Jun'ichi Fujisawa $^{2} \cdot$ Naoki Kondo $^{2}$ - Takahiro Netsu ${ }^{3}$. \\ Tadamasa Hanyu $^{3} \cdot$ Yugo Shobugawa $^{4} \cdot$ Naoto Endo $^{2}$
}

Received: 1 July 2018 / Revised: 18 February 2019 / Accepted: 26 February 2019 / Published online: 8 March 2019

(c) The Author(s) 2019

\begin{abstract}
Purpose To investigate the prevalence of and factors associated with dysfunctional low back pain (LBP) in patients with rheumatoid arthritis (RA).

Methods This cross-sectional study included 1276 RA outpatients from two hospitals. The Roland-Morris Disability Questionnaire was used to address the functional-dysfunctional state criterion. Clinical variables, such as medical status, disease activity, bone mineral density, and spinopelvic alignment parameters, were compared between patients with and without dysfunctional LBP. Results Mean age and disease duration were 64.6 and 13.4 years, respectively; the prevalence of dysfunctional LBP was $32.8 \%$. On univariate analysis, significant differences existed in many variables, except sex, body weight, C-reactive protein (CRP) level, and prevalence of biological agent users, between patients with and without dysfunctional LBP. Multivariate logistic regression analysis revealed body mass index (BMI; odds ratio [OR], 1.116; $P<0.001$ ), onset age of RA (OR, 1.020; $P=0.020)$, disease duration of RA (OR, 1.043; $P<0.001$ ), methotrexate (MTX) use (OR, 0.609; $P=0.007$ ), vertebral fractures (OR, 2.189; $P=0.001)$, vertebral endplate and/or facet erosion (OR, $1.411 ; P=0.043)$, disease activity score (DAS) in 28 joints-CRP (DAS-28CRP) (OR, 1.587; $P=0.001$ ), pelvic tilt (PT; OR, 1.023; $P=0.019)$, and sagittal vertical axis (SVA; OR, $1.007 ; P=0.043$ ) as associated factors.

Conclusion The factors associated with dysfunctional LBP in patients with RA were more vertebral fractures, higher DAS28CRP, vertebral endplate and/or facet erosion, higher BMI, longer disease duration, greater PT, older onset age, greater SVA, and less MTX use. Strictly controlling patients' body weight and disease activity with MTX and avoiding spinopelvic malalignment through vertebral fracture prevention are important.
\end{abstract}

\section{Graphical abstract}

These slides can be retrieved under Electronic Supplementary Material.
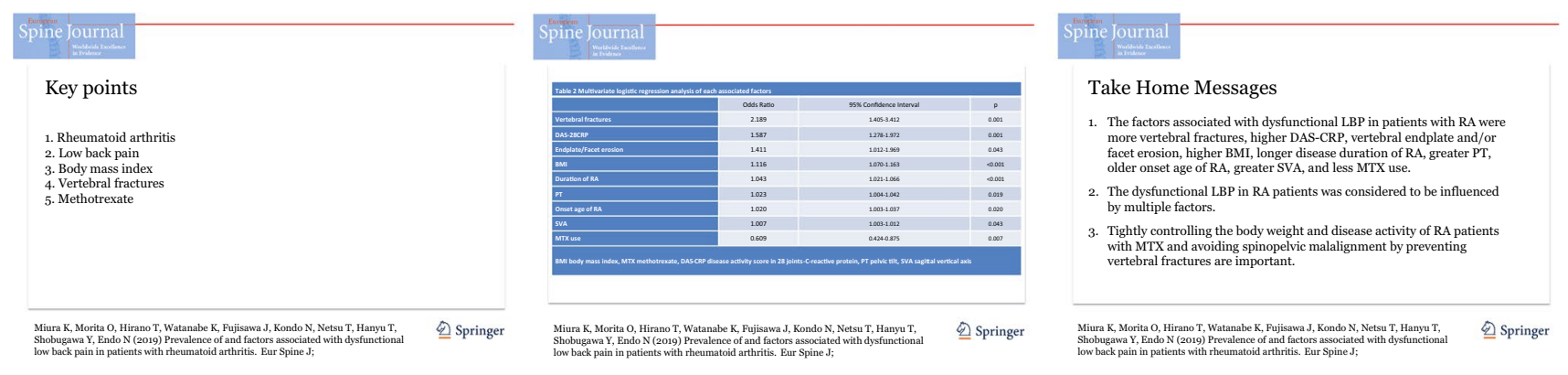

The investigation was performed at Nagaoka Red Cross Hospital and Niigata University School of Medicine, Niigata, Japan.

Electronic supplementary material The online version of this article (https://doi.org/10.1007/s00586-019-05938-x) contains supplementary material, which is available to authorized users.

Extended author information available on the last page of the article 
Keywords Rheumatoid arthritis · Low back pain · Body mass index · Spinopelvic malalignment - Vertebral fractures . Methotrexate

\section{Introduction}

It is estimated that $15 \%-20 \%$ of adults experience low back pain (LBP) during a single year and 50\%-80\% experience at least one episode of LBP during a lifetime [1]. Most LBP cases are nonspecific; however, in approximately $10 \%$ of cases, a specific cause is identified [2]. Studies showed that LBP was associated with disability and quality of life (QOL) [3, 4].

Rheumatoid arthritis (RA), the most common inflammatory disease, affects $\geq 1 \%$ of the world's population [5]. Although the prevalence, characteristics, natural course, and therapy of cervical lesions of RA have been reported and clarified because of their clinical importance, thoracolumbar lesions in RA have attracted less attention [6-9]. Studies have reported a higher prevalence of LBP with RA and indicated radiographic findings, disease activity of RA, and psychological factors are associated factors [10-12]. However, LBP in RA patients has not been evaluated in terms of lumbar function in these studies.

Assessing the dysfunctional status caused by LBP in RA patients is imperative. The present study aimed to clarify the prevalence of and the factors associated with dysfunctional LBP in RA patients based on the functional-dysfunctional criterion for LBP.

\section{Materials and methods}

\section{Study design}

This cross-sectional study was approved by an institutional research ethics board; we enrolled RA patients from the outpatient clinics of Nagaoka Red Cross Hospital and Niigata University Hospital. All patients provided written informed consent before the enrollment.

\section{Patients}

From August 2015 to March 2016, we recruited patients diagnosed with RA according to the revised American College of Rheumatology 1987 criteria [13]. The inclusion criteria were (1) available whole spinopelvic anteroposterior and lateral radiographs of standing position and (2) completion of the questionnaire on their own.

The exclusion criteria were (1) previous spine surgery and (2) inability to stand without assistance. In total, 1449 patients were initially enrolled. Of these patients, 173 were excluded because of the aforementioned criteria. Finally, 1276 patients were included.

\section{Roland-Morris Disability Questionnaire and definition of dysfunctional LBP definition}

The Roland-Morris Disability Questionnaire (RDQ) [14] was used to determine dysfunctional LBP. This self-reported outcome measure was translated and validated in Japanese in 2003 [15]. Stratford et al. [16] indicated that a threshold of $>4 / 24$ RDQ points provided a reasonably accurate classification LBP of patients as functional or dysfunctional. Hence, we defined dysfunctional LBP as $\geq 5$ RDQ points.

\section{Clinical assessment}

At the time of the survey, the following demographic characteristics, clinical variables, and current medications were investigated: age, sex, body mass index (BMI; weight $[\mathrm{kg}] /$ height $\left.{ }^{2}\left[\mathrm{~m}^{2}\right]\right)$, age at RA onset, disease duration of RA, serum level of rheumatoid factor (RF), serum level of C-reactive protein (CRP), serum level of matrix metalloprotease (MMP)-3, visual analog scale (VAS) score for LBP, disease activity score in 28 joints-CRP (DAS-28CRP), history of joint surgery, daily corticosteroid use, methotrexate (MTX) use, and biological agents (BIO) use.

\section{Radiological assessment}

The radiographical technique for measuring spinopelvic parameters was standardized as reported by Oe et al. [17]. Digital radiographs were transferred as digital imaging and communications in medicine data to a computer and measured using Surgimap Spine (Nemaris Inc., NY, USA). The measured parameters in the radiographs were as follows: (1) Cobb angle of coronal curvature; (2) pelvic tilt (PT: angle between the line connecting the midpoint of the sacral plate to the femoral head axis and the vertical axis); (3) pelvic incidence (PI: the angle between the line perpendicular to the sacral plate at its midpoint and the line connecting this point to the axis of the femoral heads); (4) pelvic incidence minus lumbar lordosis (PI-LL); and (5) sagittal vertical axis (SVA: the distance between a plumb line from the center of the C7 vertebral body and posterior superior corner of sacrum). Spondylolisthesis was assessed anteroposteriorly and laterally on radiographs. Slip measurements according to Meyerding Grading Scale Grade 1 were considered to indicate spondylolisthesis [18]. Vertebral fracture was 
assessed with lateral radiographs of the thoracolumbar spine and diagnosed by a semiquantitative visual method [19]. Scoliosis was defined as a coronal curvature of $\geq 10^{\circ}$ on anteroposterior radiographs. Intervertebral and/or facet erosion was defined according to the definition of Kawaguchi et al. [10]. Dual-energy X-ray absorptiometry of the hip joint was performed to evaluate bone mineral density (BMD) using a Hologic QDR 4500 (Hologic, Waltham, MA, USA) with the patient in a supine position.

Radiological measurements were reviewed by two experienced orthopedic spine surgeons blinded to the clinical data. To evaluate the measurements' reliability, all patients underwent measurements twice by the first (K.M.) and second (O.M.) authors. Interclass correlation coefficient values for the intraobserver and interobserver errors were 0.926-0.976 and 0.851-0.964, respectively, in all valuable measurements. Consequently, the measurement methods were considered reliable.

\section{Statistical analysis}

Baseline patient data were expressed as mean \pm standard deviation (continuous variables) or as the number (percentage; categorical variables). Data were stratified by the presence of dysfunctional LBP (RDQ $>4)$. We used the unpaired $\mathrm{t}$ test for continuous variables and Chi-square test for categorical variables to examine difference between patients with and without dysfunctional LBP. To assess the independent factors associated with dysfunctional LBP, multivariate logistic regression analysis was performed using variables that had $P<0.10$ on univariate analysis. All statistical analyses were performed using SPSS version 22.0 (IBM-SPSS, Inc., Chicago, IL, USA). $P<0.05$ was considered statistically significant.

\section{Results}

Demographic and clinical features of patients are listed in Table 1. Mean age was 64.6 years (men 19.4\%). Mean age of onset and mean disease duration were 51.1 and 13.4 years, respectively. Mean BMI was $22.5 \mathrm{~kg} / \mathrm{m}^{2}$. A total of $21.5 \%$, $73.6 \%$, and $32.1 \%$ of patients had been treated with corticosteroids, MTX, and BIO, respectively. Moreover, $27.1 \%$ had undergone joint surgery for RA. Prevalence of scoliosis, vertebral fractures, spondylolisthesis, and endplate/facet joint erosion was $35.3 \%, 13.8 \%, 21.1 \%$, and $36.3 \%$, respectively.

Dysfunctional LBP occurred in 419 of the 1276 patients (prevalence $32.8 \%$ ). On the univariate analysis, dysfunctional LBP patients were older $(P<0.001)$ and shorter $(P<0.001)$, had higher BMI $(P<0.001)$, had older age of RA onset $(P=0.001)$, had longer disease duration $(P<0.001)$, and had higher CRP level $(P=0.017)$. Patients with dysfunctional LBP more frequently used corticosteroid $(P<0.001)$ and less frequently used MTX $(P<0.001)$. Patients with dysfunctional LBP had a more frequent history of joint surgery $(P=0.001)$. DAS-28CRP was significantly higher in patients with dysfunctional LBP than in those without it $(P<0.001)$. Prevalence of thoracolumbar lesions (scoliosis, vertebral fractures, spondylolisthesis, and endplate and/or facet erosion) was significantly higher in dysfunctional LBP patients $(P<0.001,0.001,0.001$, and 0.001 , respectively). All values of spinopelvic parameter values were significantly worse in dysfunctional LBP patients $(P<0.001)$. BMD was significantly lower in dysfunctional LBP patients $(P<0.001)$.

Multivariate analysis revealed that vertebral fractures (odds ratio [OR], 2.128; 95\% confidence interval [CI], $1.343-3.371 ; P=0.001$ ), DAS-28CRP (OR, 1.587; 95\% CI, 1.278-1.972; $P=0.001)$, endplate and/or facet erosion (OR, 1.411; 95\% CI, 1.012-1.969; $P=0.043$ ), BMI (OR, 1.116; 95\%, CI, $1.070-1.163 ; P<0.001)$, disease duration of RA (OR, 1.043; 95\% CI, 1.021-1.066; $P<0.001$ ), PT (OR, $1.023 ; 95 \% \mathrm{CI}, 1.004-1.042 ; P=0.019$ ), age of RA onset (OR, $1.020 ; 95 \% \mathrm{CI}, 1.003-1.037 ; P=0.020)$, SVA (OR; $1.007 ; 95 \%$ CI, $1.003-1.012, P=0.043$ ), and MTX use (OR, 0.609; 95\% CI, 0.424-0.875; $P=0.007$ ) were factors associated with dysfunctional LBP (Table 2).

\section{Discussion}

Rheumatoid granulomatous nodule of the lumbar spine was reported for the first time as a lumbar lesion with rheumatoid arthritis by Baggenstoss et al. [20]. Then, disk narrowing without osteophytes, spondylolisthesis, facet joint erosion, and osteoporosis were indicated to be distinct radiological features of the lumbar spine in RA patients [21]. Previous reports have also demonstrated a higher prevalence of LBP associated with the aforementioned lumbar lesions in RA patients [12, 22]. In addition to their peripheral joint lesions, RA patients with LBP had a significantly higher degree of disability and lower quality of life than did those without LBP, as shown by Kothe et al. [12]. Baykara et al. [22] also indicated that the association of RA with LBP causes a significant decrease in patients' functional capacity and QOL. However, these reports have not mentioned the threshold value to classify LBP patients as functional or dysfunctional. It is important to clarify the factors associated with dysfunctional LBP based on the clear threshold value of dysfunctional LBP in RA patients. Stratford et al. estimated a threshold target RMQ value that could be used to classify 143 patients with LBP as functional or dysfunctional and found that a threshold value of 4 RMQ points provided a reasonably accurate classification of patients [16]. Its sensitivity 
Table 1 Demographic and clinical features of the study population

\begin{tabular}{|c|c|c|c|c|}
\hline & Total & $\begin{array}{l}\text { Dysfunctional LBP } \\
\text { RDQ > } 4(\mathrm{n}=419)\end{array}$ & $\begin{array}{l}\text { Functional LBP } \\
\text { RDQ } \leq 4(n=857)\end{array}$ & $p$ \\
\hline Age (years) & $64.6 \pm 11.9$ & $68.7 \pm 11.2$ & $62.5 \pm 11.7$ & $<0.001 *$ \\
\hline Female $(\%)$ & $1028(80.6)$ & $342(81.6)$ & $686(80.0)$ & $\left.0.547^{\dagger}\right)$ \\
\hline $\mathrm{BH}(\mathrm{m})$ & $155.3 \pm 8.2$ & $153.5 \pm 8.8$ & $156.4 \pm 7.7$ & $<0.001 *$ \\
\hline BW (kg) & $54.4 \pm 10.7$ & $55.2 \pm 11.8$ & $54.1 \pm 10.0$ & $0.123^{*}$ \\
\hline BMI $\left(\mathrm{kg} / \mathrm{m}^{2}\right)$ & $22.5 \pm 3.8$ & $23.4 \pm 4.3$ & $22.0 \pm 3.4$ & $<0.001 *$ \\
\hline Onset age of RA (years) & $51.1 \pm 13.9$ & $53.0 \pm 13.9$ & $50.2 \pm 13.8$ & $0.001 *$ \\
\hline Duration of RA (years) & $13.4 \pm 10.4$ & $15.7 \pm 11.2$ & $12.2 \pm 9.8$ & $<0.001 *$ \\
\hline RF (IU/L) & $86.8 \pm 149.1$ & $101.0 \pm 172.2$ & $79.7 \pm 135.8$ & $0.058^{*}$ \\
\hline MMP-3 (ng/mL) & $95.3 \pm 218.3$ & $103.1 \pm 104.4$ & $91.5 \pm 255.5$ & $0.297 *$ \\
\hline $\mathrm{CRP}(\mathrm{mg} / \mathrm{dL})$ & $0.44 \pm 0.98$ & $0.55 \pm 1.38$ & $0.38 \pm 0.70$ & $0.017 *$ \\
\hline Corticosteroid use (\%) & $274(21.5)$ & $119(28.4)$ & $155(18.1)$ & $<0.001^{\dagger}$ \\
\hline MTX use $(\%)$ & 939 (73.6) & $281(67.1)$ & $658(76.8)$ & $<0.001^{\dagger}$ \\
\hline BIO use (\%) & $413(32.4)$ & $147(35.1)$ & $266(31.0)$ & $0.161^{\dagger}$ \\
\hline DAS-28CRP & $2.15 \pm 0.76$ & $2.39 \pm 0.76$ & $2.04 \pm 0.73$ & $<0.001^{*}$ \\
\hline History of joint surgery (\%) & $346(27.1)$ & $150(35.8)$ & $196(22.9)$ & $0.001^{\dagger}$ \\
\hline Scoliosis $\left(\mathrm{Cobb}>10^{\circ}\right)(\%)$ & $451(35.3)$ & $199(47.6)$ & $252(29.4)$ & $<0.001^{\dagger}$ \\
\hline Vertebral fractures $(\%)$ & $176(13.8)$ & $111(26.6)$ & $65(7.6)$ & $<0.001^{\dagger}$ \\
\hline Spondylolisthesis (\%) & $269(21.1)$ & $118(28.2)$ & $151(17.6)$ & $<0.001^{\dagger}$ \\
\hline Endplate/facet erosion (\%) & $463(36.3)$ & $210(50.6)$ & $253(29.6)$ & $<0.001^{\dagger}$ \\
\hline $\mathrm{PT}\left({ }^{\circ}\right)$ & $17.8 \pm 10.1$ & $22.1 \pm 12.0$ & $15.8 \pm 8.3$ & $<0.001 *$ \\
\hline PI-LL $\left(^{\circ}\right)$ & $6.1 \pm 16.0$ & $13.2 \pm 20.5$ & $2.7 \pm 11.9$ & $<0.001 *$ \\
\hline SVA (mm) & $20.5 \pm 46.6$ & $42.0 \pm 59.1$ & $10.1 \pm 34.7$ & $<0.001^{*}$ \\
\hline BMD of hip joint $\left(\mathrm{g} / \mathrm{cm}^{2}\right)$ & $0.609 \pm 0.125$ & $0.592 \pm 0.135$ & $0.617 \pm 0.118$ & $<0.001^{*}$ \\
\hline
\end{tabular}

$R D Q$ The Roland-Morris Disability Questionnaire, $B M I$ body mass index, $R F$ rheumatoid factor, $M M P$ 3 matrix metalloproteinase-3, CRP C-reactive protein, $M T X$ methotrexate, $B I O$ biological agents, DAS$28 C R P$ disease activity score in 28 joints-C-reactive protein, $P T$ pelvic tilt, $P I-L L$ pelvic incidence minus lumbar lordosis, $S V A$ sagittal vertical axis, $B M D$ bone mineral density

Data were expressed as mean \pm standard deviation (continuous variables) or as number (percentage; categorical variables)

*Unpaired $t$ test

${ }^{\dagger}$ Chi-square test

Table 2 Multivariate logistic regression analysis of each associated factor

\begin{tabular}{lllr}
\hline & Odds ratio & $\begin{array}{l}\text { 95\% Confidence } \\
\text { interval }\end{array}$ & $p$ \\
\hline Vertebral fractures & 2.189 & $1.405-3.412$ & 0.001 \\
DAS-28CRP & 1.587 & $1.278-1.972$ & 0.001 \\
Endplate/facet erosion & 1.411 & $1.012-1.969$ & 0.043 \\
BMI & 1.116 & $1.070-1.163$ & $<0.001$ \\
Duration of RA & 1.043 & $1.021-1.066$ & $<0.001$ \\
PT & 1.023 & $1.004-1.042$ & 0.019 \\
Onset age of RA & 1.020 & $1.003-1.037$ & 0.020 \\
SVA & 1.007 & $1.003-1.012$ & 0.043 \\
MTX use & 0.609 & $0.424-0.875$ & 0.007 \\
\hline
\end{tabular}

$D A S$ - $C R P$ disease activity score in 28 joints-C-reactive protein, $B M I$ body mass index, $P T$ pelvic tilt, $S V A$ sagittal vertical axis, $M T X$ methotrexate and specificity for a threshold score of 4 were $94 \%$ and $69 \%$, respectively.

The present study demonstrated that the prevalence of dysfunctional LBP in 1276 Japanese RA patients was $32.8 \%$. To our knowledge, this is the first report that clarified the prevalence of and the independent factors associated with LBP that contribute to dysfunction in RA patients determined using health-related QOL measure. Concerning the aforementioned threshold of functional LBP using RDQ, the prevalence of dysfunctional LBP in the general Japanese population (mean age, 53.4 [21-86] years) was reported to be $15 \%-20 \%$ [23]. Thus, the prevalence of dysfunctional LBP in RA patients may be higher than that of dysfunctional LBP in the general population.

On the multivariate analysis, vertebral fractures, DAS28CRP, endplate and/or facet erosion, BMI, duration of RA, PT, age of RA onset, SVA, and MTX were the independent factors associated with dysfunctional LBP. Vertebral 
fractures had the greatest OR among the factors influencing dysfunctional LBP in this study. Kuroda et al. [24] indicated that back pain was significantly associated with osteoporosis in 899 Japanese postmenopausal women with RA. Omata et al. [25] demonstrated that vertebral fractures were associated with reduced functional status in 200 postmenopausal RA patients. Furthermore, PT and SVA were also associated with dysfunctional LBP in this study. Sagittal spinopelvic parameters (SVA and PT) and mismatch between LL and PI (PI-LL) have strong correlations with disability and QOL of adult patients with spinal deformity [26]. Yokoyama et al. [27] reported that patients with vertebral fractures had significantly longer SVA than did their healthy counterparts. Thus, vertebral fractures themselves and spinopelvic malalignment with or without vertebral fractures might deteriorate QOL with back pain and lumbar dysfunction.

DAS-28CRP and endplate/facet erosion were other factors associated with dysfunctional LBP following vertebral fractures in terms of odds ratio in this study. In a Japanese cross-sectional study of RA patients, Yamada et al. [11] demonstrated that the factor most strongly associated with severe LBP was a high DAS28, and they hypothesized that inflammatory response in lumbar spine secondary to high RA activity results in severe LBP. van der Heijde et al. [28] suggested that greater radiographic damage and progression correlate with a higher degree of disability in RA patients. Kawaguchi et al. [10] analyzed the radiological findings of the lumbar spine and clinical symptoms in 106 RA patients: 42 patients (40\%) had symptoms of LBP, and endplate erosion was observed. Dysfunction with severe LBP might be associated with high disease activity and erosion.

Obesity is an important independent predictor of back pain [29], and a similar result was obtained in RA patients in this study. Late onset of RA was associated with dysfunctional LBP. Tan et al. [30] analyzed 1206 RA patients and described that elderly onset RA patients had a higher number of RA-related comorbidities and poorer functional status than their counterparts. Ruban et al. [31] showed that late-onset RA patients have greater disease activity that may contribute to disability early in the disease course. These differences in clinical characteristics between young and elderly onset of RA can affect lumbar function.

Disease duration and MTX use were independent associated factors, whereas age was not, suggesting that pathological changes in lumbar spine by RA are more responsible for dysfunctional LBP compared to degenerative changes by aging. The mean disease duration of the dysfunctional LBP group was 15.7 years, indicating that the mean onset time of RA was from 1999 to 2000; these years were timed to coincide with MTX initiation for RA in Japan. Thus, patients with dysfunctional LBP might have less chance to be treated with MTX at the onset of RA compared to those without dysfunctional LBP. Although initial treatment in each patient could not be surveyed in this study, patients with dysfunctional LBP less frequently used MTX when this study was conducted. Therefore, strict disease control from the early-onset period of RA using MTX might be important in preventing dysfunctional LBP. Moreover, those patients without MTX treatment at disease onset could receive corticosteroid more frequently, resulting in increased vertebral fracture risk.

This study has several limitations. First, we did not evaluate the influence of psychological factors. Among the various complications of RA, depression is the most common, affecting approximately $15 \%$ of RA patients [32]. Studies have indicated the independent relationship between depressive symptoms and decreased general physical or mental health (QOL) in RA patients $[33,34]$. Kothe et al. [12] reported a significantly higher degree of disability and depression in RA patients with LBP than in those without LBP. LBP is an important factor for the physical and psychological behaviors of RA patients. Second, we evaluated the surgical history of joints but not the joint lesions and lower extremity alignment. Several studies indicated that hip lesion and leg length alignment had played an important role in the occurrence of LBP in non-RA patients [35-37]. RA affects all synovial joints, causing painful swelling that can cause bone erosion, joint deformity, and malalignment. Third, the dysfunctional threshold of RDQ $>4$ was provided by a report in the general population, not in RA patients. Thus, it is unknown whether this criterion is applicable to RA patients.

In conclusion, this study revealed that the factors associated with dysfunctional LBP in RA patients were more vertebral fractures, higher DAS-28CRP, vertebral endplate and/or facet erosion, higher BMI, longer disease duration, greater PT, older onset age, greater SVA, and less MTX use. The dysfunctional LBP in RA patients was considered to be influenced by multiple factors. Strict control of the body weight and disease activity in RA patients with MTX and avoiding spinopelvic malalignment by preventing vertebral fractures are important.

\section{Compliance of ethical standards}

Conflict of interest None of the authors have any potential conflict of interest.

Informed consent We declare that all patients gave informed consent prior to inclusion in this study.

Ethical approval This cross-sectional study was approved by an institutional research ethics board; we enrolled RA patients from the outpatient clinics of Nagaoka Red Cross Hospital and Niigata University Hospital. All patients provided written informed consent before the enrollment. 
Open Access This article is distributed under the terms of the Creative Commons Attribution 4.0 International License (http://creativeco mmons.org/licenses/by/4.0/), which permits unrestricted use, distribution, and reproduction in any medium, provided you give appropriate credit to the original author(s) and the source, provide a link to the Creative Commons license, and indicate if changes were made.

\section{References}

1. Rubin DI (2007) Epidemiology and risk factors for spine pain. Neurol Clin 25:353-371. https://doi.org/10.1016/j. ncl.2007.01.004

2. Krismer M, van Tulder M, Low Back Pain Group of the Bone and Joint Health Strategies for Europe Project (2007) Strategies for prevention and management of musculoskeletal conditions. Low back pain (non-specific). Best Pract Res Clin Rheumatol 21:77-91. https://doi.org/10.1016/j.berh.2006.08.004

3. Horng YS, Hwang YH, Wu HC et al (2005) Predicting healthrelated quality of life in patients with low back pain. Spine (Phila Pa 1976) 30:551-555

4. Silverman SL, Piziak VK, Chen P et al (2005) Relationship of health related quality of life to prevalent and new or worsening back pain in postmenopausal women with osteoporosis. J Rheumatol 32:2405-2409

5. Lawrence RC, Helmick CG, Arnett FC et al (1998) Estimates of the prevalence of arthritis and selected musculoskeletal disorders in the United States. Arthritis Rheum 41:778-799. https://doi.org/10.1002/1529-0131(199805)41:5\%3c778 :AID-ART4\%3e3.0.CO;2-V

6. Yurube T, Sumi M, Nishida K et al (2011) Progression of cervical spine instabilities in rheumatoid arthritis: a prospective cohort study of outpatients over 5 years. Spine (Phila Pa 1976) 36:647653. https://doi.org/10.1097/BRS.0b013e3181da21c5

7. Boden SD (1994) Rheumatoid arthritis of the cervical spine. Surgical decision making based on predictors of paralysis and recovery. Spine (Phila Pa 1976) 19:2275-2280

8. Kaito T, Ohshima S, Fujiwara $\mathrm{H}$ et al (2013) Predictors for the progression of cervical lesion in rheumatoid arthritis under the treatment of biological agents. Spine (Phila Pa 1976) 38:22582263. https://doi.org/10.1097/BRS.0000000000000066

9. Takahashi S, Suzuki A, Koike T et al (2014) Current prevalence and characteristics of cervical spine instability in patients with rheumatoid arthritis in the era of biologics. Mod Rheumatol 24:904-909. https://doi.org/10.3109/14397595.2014.895123

10. Kawaguchi Y, Matsuno H, Kanamori M et al (2003) Radiologic findings of the lumbar spine in patients with rheumatoid arthritis, and a review of pathologic mechanisms. J Spinal Disord Tech $16: 38-43$

11. Yamada K, Suzuki A, Takahashi S et al (2015) Severe low back pain in patients with rheumatoid arthritis is associated with disease activity score but not with radiological findings on plain X-rays. Mod Rheumatol 25:56-61. https://doi.org/10.3109/14397 595.2014.924187

12. Kothe R, Kohlmann T, Klink T et al (2007) Impact of low back pain on functional limitations, depressed mood and quality of life in patients with rheumatoid arthritis. Pain 127:103-108. https:// doi.org/10.1016/j.pain.2006.08.011

13. Arnett FC, Edworthy SM, Bloch DA et al (1988) The American Rheumatism Association 1987 revised criteria for the classification of rheumatoid arthritis. Arthritis Rheum 31:315-324

14. Roland M, Richard M (1983) A study of the natural history of back pain: part I: development of a reliable and sensitive measure of disability in low-back pain. Spine (Phila Pa 1976) 8:141-144
15. Nakamura M, Miyamoto K, Shimizu K (2003) Validation of the Japanese version of the Roland-Morris Disability Questionnaire for Japanese patients with lumbar spinal diseases. Spine (Phila Pa 1976) 28:2414-2418. https://doi.org/10.1097/01.BRS0000085 425.90995 .09

16. Stratford PW, Riddle DL (2016) A Roland Morris Disability Questionnaire target value to distinguish between functional and dysfunctional status in people with low back pain. Physiotherapy Canada 68:29-35. https://doi.org/10.3138/ptc.2014-85

17. Oe S, Togawa D, Nakai K et al (2015) The influence of age and sex on cervical alignment among volunteers aged over 50 . Spine (Phila Pa 1976) 40:1487-1494. https://doi.org/10.1097/ BRS.0000000000001071

18. Meyereding HW (1932) Spondylolisthesis. Surg Gynecol Obstet 54:371-379

19. Genant HK, Jergas M, Palermo L et al (1996) Comparison of semiquantitative visual and quantitative morphometric assessment of prevalent and incident vertebral fractures in osteoporosis: the study of osteoporotic fractures research group. J Bone Miner Res 11:984-996. https://doi.org/10.1002/jbmr.5650110716

20. Baggenstoss AH, Bickel WH, Ward LE (1952) Rheumatoid granulomatous nodules as destructive lesions. J Bone Joint Surg Am 34:601-609

21. Lawrence JS, Sharp J, Ball J et al (1964) Rheumatoid arthritis of the lumbar spine. Ann Rheum Dis 23:205-217

22. Baykara RA, Bozgeyik Z, Akgul O et al (2013) Low back pain in patients with rheumatoid arthritis: clinical characteristics and impact of low back pain on functional ability and health related quality of life. J Back Musculoskelet Rehabil 26:367-374. https ://doi.org/10.3233/BMR-130393

23. Suzukamo Y (2009) Outcome assessment by Roland-Morris Disability Questionnaire (RDQ). J Lumbar Spine Disord 15:17-22 (in Japanese)

24. Kuroda T, Shiraki M, Tanaka S et al (2009) The relationship between back pain and future vertebral fracture in postmenopausal women. Spine (Phila Pa 1976) 34:1984-1989. https://doi. org/10.1097/BRS.0b013e3181b0c97a

25. Omata Y, Hagiwara F, Nishino J et al (2014) Vertebral fractures affect functional status in postmenopausal rheumatoid arthritis patients. J Bone Miner Metab 32:725-731. https://doi. org/10.1017/s00774-013-0552-8

26. Glassman SD, Bridwell K, Dimar JR et al (2005) The impact of positive sagittal balance in adult spinal deformity. Spine (Phila Pa 1976) 30:2024-2029

27. Yokoyama K, Kawanishi M, Yamada M et al (2015) Postoperative change in sagittal balance after kyphoplasty or the treatment of osteoporotic vertebral compression fracture. Eur Spine J 24:744749. https://doi.org/10.1007/s00586-014-3678-Z

28. van der Heijde D, Landewe R, van Vollenhoven R et al (2008) Level of radiographic damage and radiographic progression are determinants of physical function: a longitudinal analysis of the TEMPO trial. Ann Rheum Dis 67:1267-1270. https://doi. org/10.1136/ard.2007.081331

29. Webb R, Brammah T, Lunt M et al (2003) Prevalence and predictors of intense, chronic, and disabling neck and back pain in the UK general population. Spine (Phila Pa 1976) 28:1195-1202. https://doi.org/10.1097/BRS.0000067430.49169.01

30. Tan TC, Gao X, Thong BY et al (2017) Comparison of elderly- and young-onset rheumatoid arthritis in an Asian cohort. Int J Rheum Dis 20:737-745. https://doi.org/10.1111/1756-185X.12861

31. Ruban TN, Jacob B, Pope JE et al (2016) The influence of age at disease onset on disease activity and disability: results from the Ontario Best Practices Research Initiative. Clin Rheumatol 35:759-763. https://doi.org/10.1007/s10067-015-3031-x

32. Dougados M, Soubrier M, Antunez A et al (2014) Prevalence of comorbidities in rheumatoid arthritis and evaluation of their 
monitoring: results of an international, cross-sectional study (COMORA). Ann Rheum Dis 73:62-68. https://doi.org/10.1136/ annrheumdis-2013-204223

33. Alishiri GH, Bayat N, Ashtiani AF et al (2008) Logistic regression models for predicting physical and mental health-related quality of life in rheumatoid arthritis patients. Mod Rheumatol 18:601-608. https://doi.org/10.3109/s10165-008-0092-6

34. Uhm DC, Nam ES, Lee HY et al (2012) Health-related quality of life in Korean patients with rheumatoid arthritis: association with pain, disease activity, disability in activities of daily living and depression. J Korean Acad Nur. 42:434-442

35. Knutson GA (2002) Incidence of foot rotation, pelvic crest unleveling, and supine leg length alignment asymmetry and their relationship to self-reported back pain. J Manipulative Physiol Ther 25:110E
36. Piazzolla A, Solarino G, Bizzoca D et al (2017) Spinopelvic parameter changes and low back pain improvement due to femoral neck anteversion in patients with severe unilateral primary hip osteoarthritis undergoing total hip replacement. Eur Spine J. https ://doi.org/10.1007/s00586-17-5033-7

37. Mochizuki T, Momohara S, Yano K et al (2016) Scoliosis related factors in patients with rheumatoid arthritis: a cross-sectional study. Mod Rheumatol 26:352-357. https://doi.org/10.3109/14397 595.2015.1097016

Publisher's Note Springer Nature remains neutral with regard to jurisdictional claims in published maps and institutional affiliations.

\section{Affiliations}

\section{Kazuto Miura ${ }^{1} \cdot$ Osamu Morita ${ }^{1}$. Toru Hirano ${ }^{2} \cdot$ Kei Watanabe $^{2}$. Jun'ichi Fujisawa ${ }^{2} \cdot$ Naoki Kondo $^{2}$. Takahiro Netsu ${ }^{3}$. Tadamasa Hanyu ${ }^{3} \cdot$ Yugo Shobugawa ${ }^{4} \cdot$ Naoto Endo $^{2}$}

\section{Kazuto Miura}

kamiu@mac.com

1 Department of Spine and Spinal Cord Surgery, Nagaoka Red Cross Hospital, 2-297-1, Senshu, Nagaoka-City, Niigata 9402108, Japan

2 Division of Orthopaedic Surgery, Department of Regenerative and Transplant Medicine, Niigata University
Graduate School of Medical and Dental Sciences, Niigata, Japan

3 Department of Rheumatology, Nagaoka Red Cross Hospital, Nagaoka, Japan

4 Division of International Health, Niigata University Graduate School of Medical and Dental Sciences, Niigata, Japan 